\title{
Relationship between Hotspots and Mantle Structure: Correlation with
}

\author{
Whole Mantle Seismic Tomography \\ Sharon Kedar, Don L. Anderson, David J. Stevenson (CalTech)
}

\begin{abstract}
We examine the relation between the locations of hotspots on the surface of the Earth and mantle structure as it appears from seismic tomography. In particular, we correlated the hotspots field with Tanimoto's 1989 shear wave velocity structure throughout the mantle, which is represented in spherical harmonics. A spherical harmonics representation of both fields enables us to perform a "degree by degree" correlation, and so to test the possible relations between features of the same scale in them. A statistical significance analysis is applied to these results. A similar analysis was performed by Richards, Hager and Sleep (1988) in studying the hotspots geoid relations.
\end{abstract}

After correlating the hotspots with Tanimoto's model, two major phenomena were observed: 1 . Very good correlation between the hotspots and slow (hot) regions in degree 2 in the bottom half of the lower mantle, which decays in the upper mantle (figure 1).

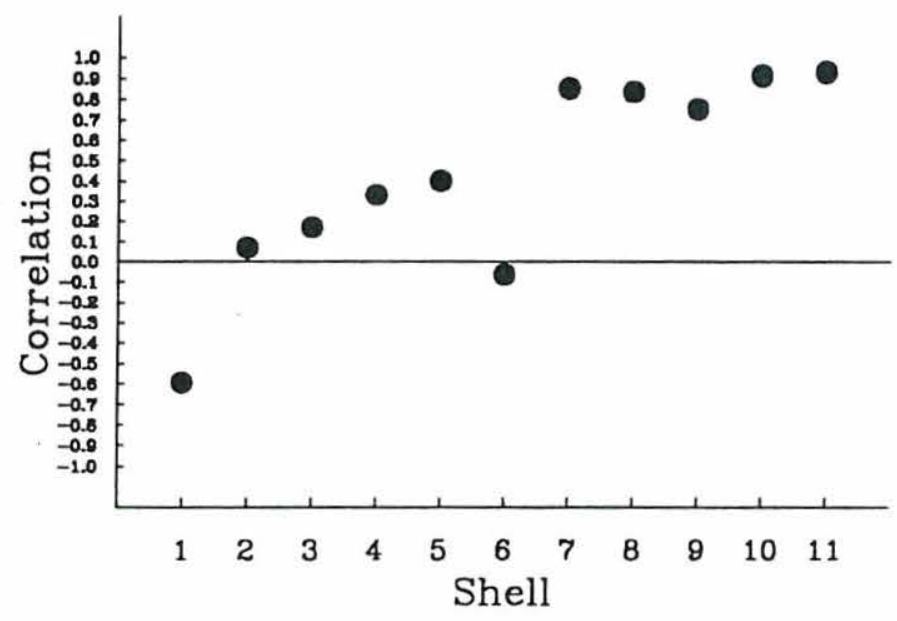

Figure 1: Variation of $\mathrm{l}=2$ Correlation from the top of the mantle (shell 1) to the bottom (shell 11) 
2. A good correlation in degree 6 in the deeper upper mantle $(200-670 \mathrm{Km})$ and lack of such correlation below $670 \mathrm{Km}$ (figure 2). These positive correlations are statistically significant (higher than $95 \%$ confidence level).

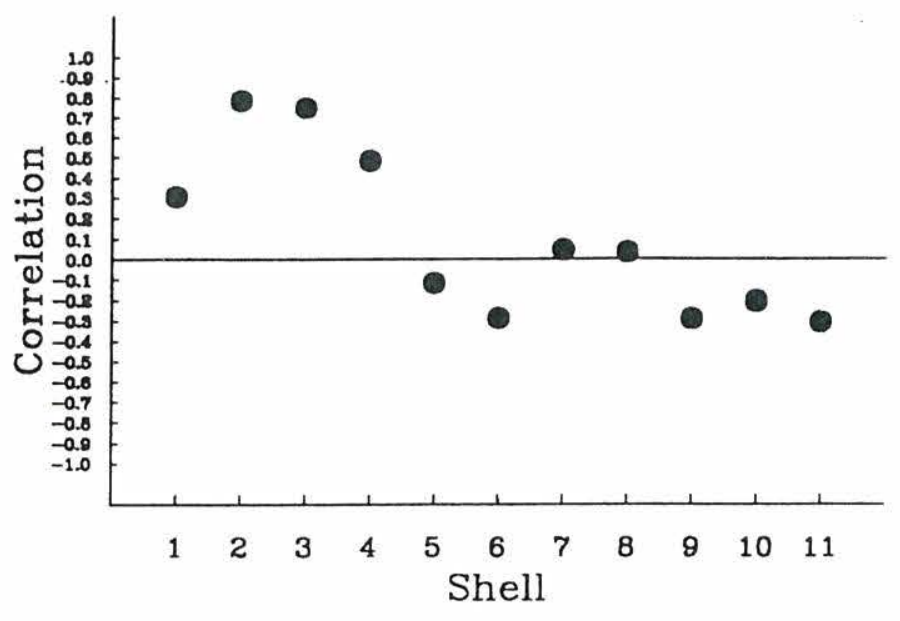

Figure 2: Variation of $\mathrm{l}=6$ Correlation from the top of the mantle (shell 1) to the bottom (shell 11)

Moreover, they are aiso a peak in the amplitude spectrum. In other words, they describe a significant part of the hotspots field. The $\mathrm{l}=2$ expansions of the lower mantle, and the $\mathrm{l}=6$ expansion of the upper mantle are presented in figures 3 and 4 together with the corresponding expansions of the hotspots field.

The key to a better understanding of the resulting correlations lies not only in the good correlations but in the lack of them. The observations made here could suggest the possibility of two separate regions in the mantle which control different parts of the hotspots field. To check this possibility we excluded those hotspots which give a negative contribution to the $l=6$ correlation in the upper mantle. The correlation of these excluded hotspots with the lower mantle gives a significant correlation for degree 2, which suggests we should look further in this direction. 
Hotspots Locations $\quad 1=2$



Lower Mantle S-Velocity $\quad \mathrm{l}=2$

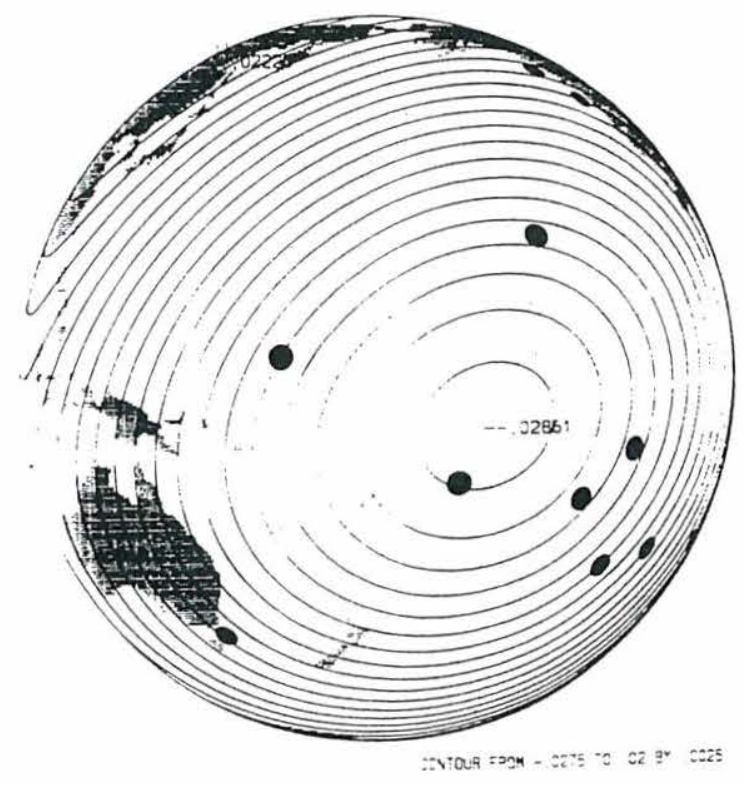

Hotspots Locations $l=2$

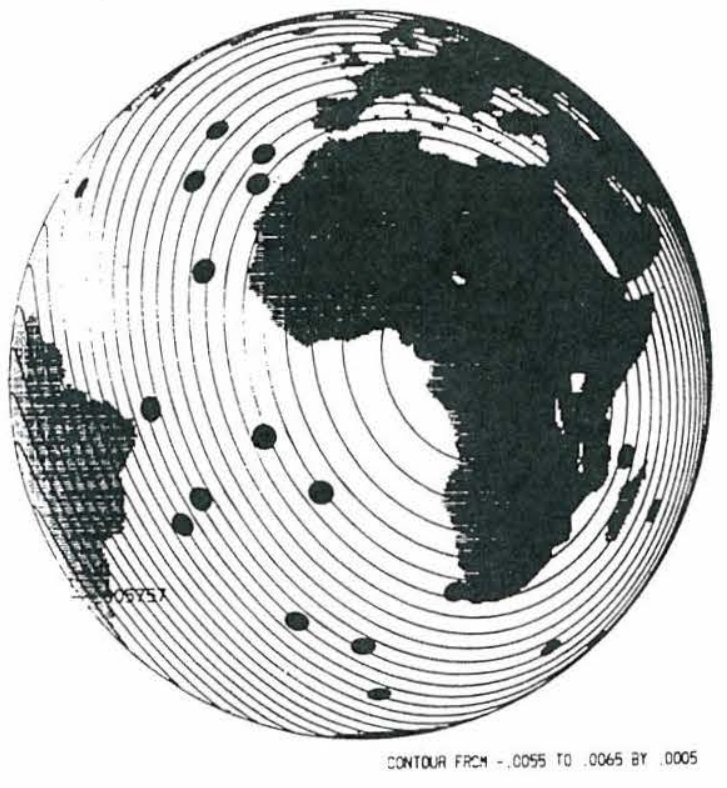

Lower Mantle S-Velocity $\mathrm{l}=2$

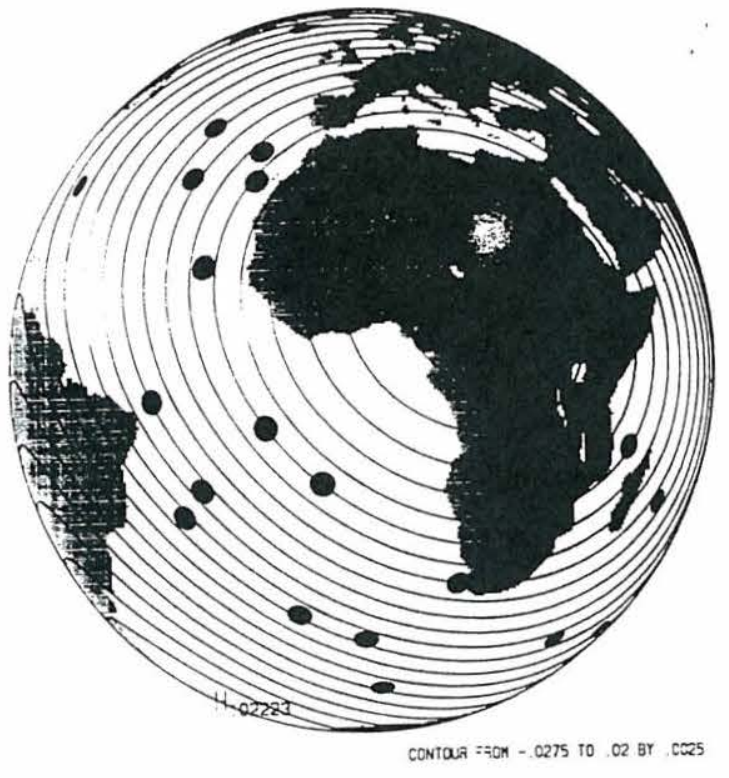


Hotspots Locations $\quad 1=6$

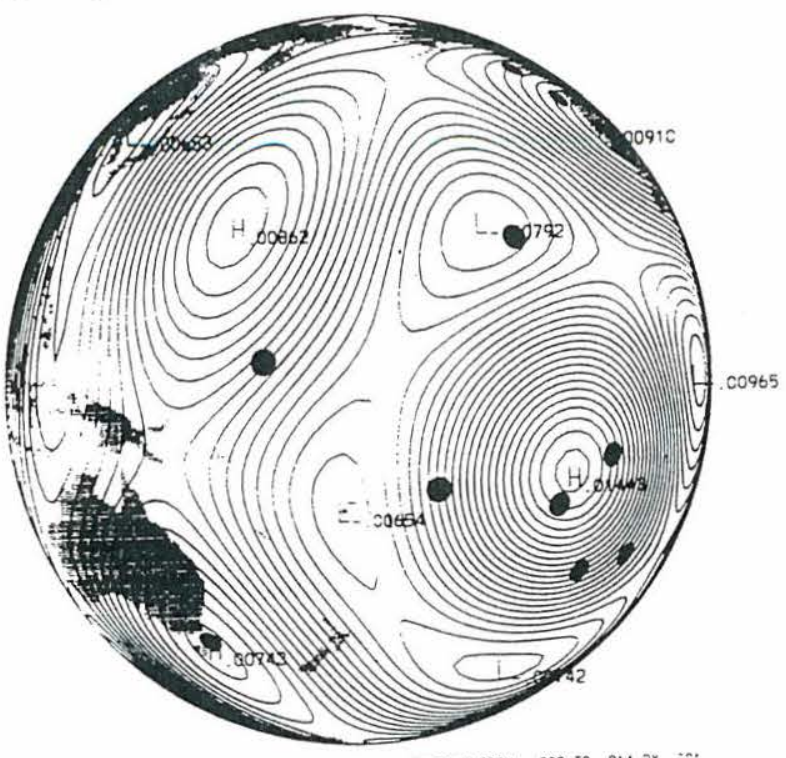

jpper Mantle $S$-Velocity $\quad l=6$

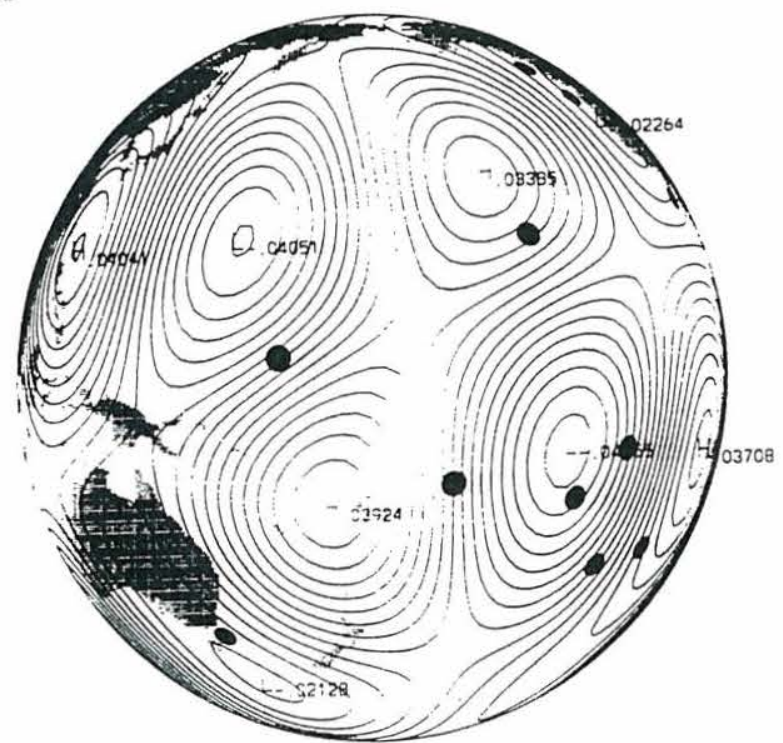

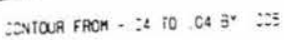

Holspots Locations $\quad l=6$

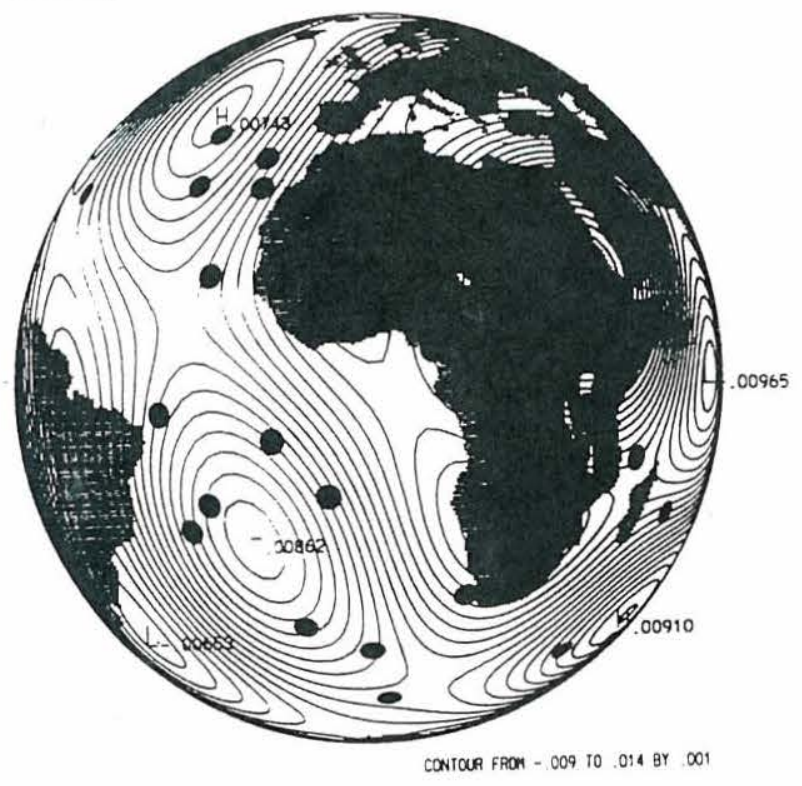

Upper Mantle S-Velocity $\quad \mathrm{l}=6$

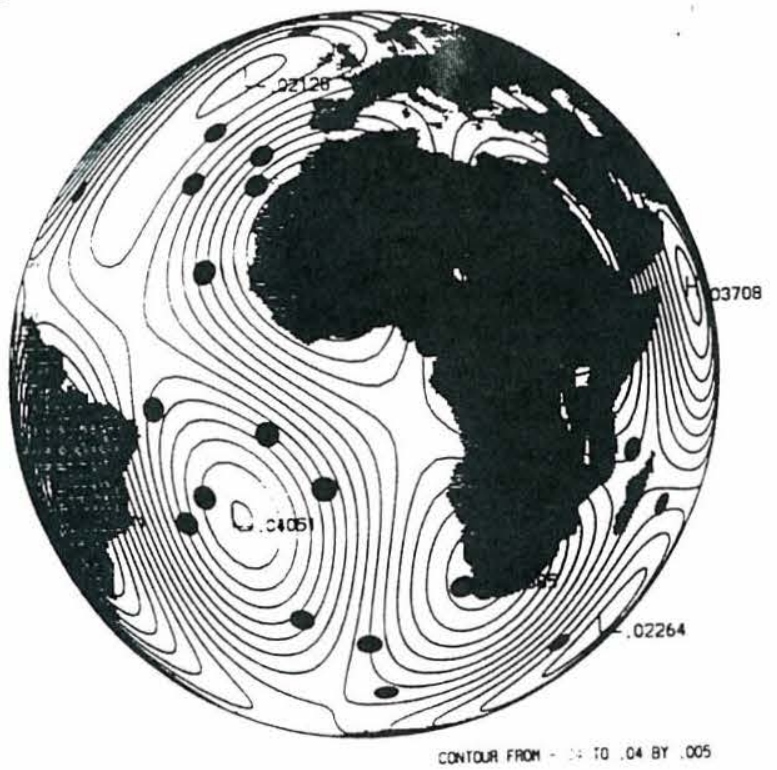

figure 4 
QE

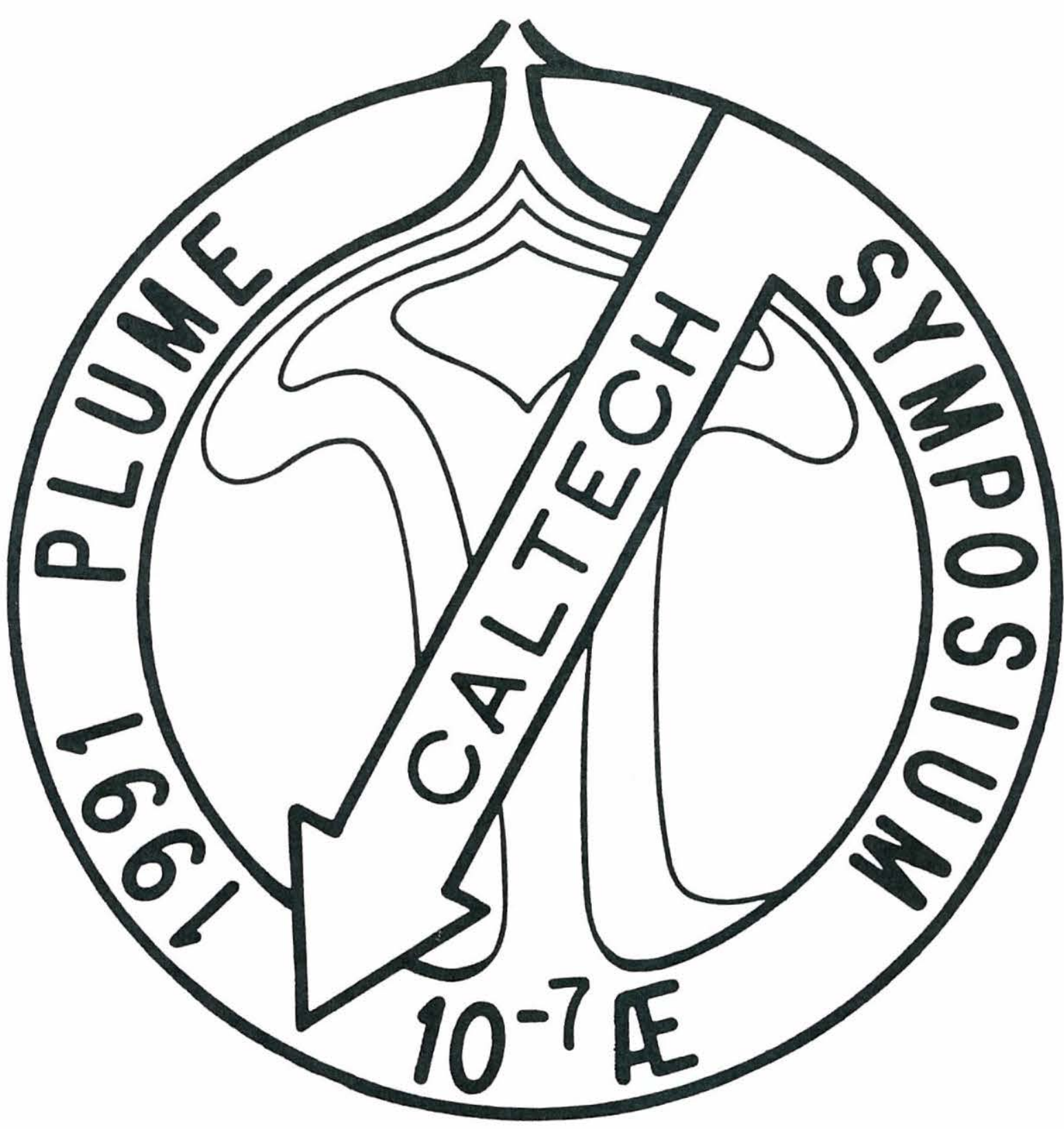

6502 